\title{
Quenched Approximation Artifacts: A study in 2-dimensional QED
}

\author{
W. Bardeen ${ }^{1}$, A. Duncan ${ }^{2}$, E. Eichten ${ }^{1}$, and H. Thacker ${ }^{3}$ \\ ${ }^{1}$ Fermilab, P.O. Box 500, Batavia, IL 60510 \\ ${ }^{2}$ Dept. of Physics and Astronomy, University of Pittsburgh, Pittsburgh, PA 15620 \\ ${ }^{3}$ Dept.of Physics, University of Virginia, Charlottesville, VA 22901
}

\begin{abstract}
The spectral properties of the Wilson-Dirac operator in 2-dimensional QED responsible for the appearance of exceptional configurations in quenched simulations are studied in detail. The mass singularity structure of the quenched functional integral is shown to be extremely compicated, with multiple branch points and cuts. The connection of lattice topological charge and exactly real eigenmodes is explored using cooling techniques. The lattice volume and spacing dependence of these modes is studied, as is the effect of clover improvement of the action. A recently proposed modified quenched approximation is applied to the study of meson correlators, and the results compared with both naive quenched and full dynamical calculations of the same quantity.
\end{abstract}




\section{Introduction}

A basic quantity in essentially all lattice calculations of hadronic properties is the quark propagator in the presence of a fixed external gauge field. Hadron propagation amplitudes or current matrix elements are extracted from averages of products of quark propagators over ensembles of gauge fields generated relative to either a pure gluonic weight $e^{\text {(pure gauge action) }}$ for the quenched approximation, or $e^{\text {(pure gauge action) }}$. (determinant of Dirac operator) for a fully dynamical calculation including internal quark loop effects. There has naturally been considerable attention devoted to the issue of the extent to which the quenched approximation deviates from the full theory [1]. Are the differences of a detailed quantitative character only, perhaps mainly absorbable in a redefinition of the scale of the theory, or are there serious qualitative differences which make it difficult to extract reliable physics from a quenched calculation?

Qualitative differences which distinguish the quenched theory from the full dynamics concern the sensitive dependence of certain amplitudes on the quark mass $m$ in the chiral limit $m \rightarrow 0$. Generic effects include the "hairpin" diagrams which signal the generation of the singlet $\eta^{\prime}$ mass and the corresponding "quenched chiral logs" [2] which are expected to give logarithmic corrections to the mass dependence in the chiral limit. In lattice calculations, finite volume effects and convergence problems in evaluating the light quark propagators are also elements which impact the study of the chiral limit. A particular artifact associated with quenched calculations involving light Wilson fermions is the occasional appearance of "exceptional" gauge configurations [3] with apparently nonsensical values for the light quark propagators resulting in extremely noisy statistical averages and unreliable values for the hadronic amplitudes. Clearly, it will be impossible to determine, in any definitive way, the chiral structure 
of the quenched theory until the nature of the exceptional configuration problem is fully understood and the resulting pathologies tamed. Our objective here is to study, in a specific model - 2 dimensional QED- under very precise numerical control, the role of the exceptional configuration phenomenon in quenched calculations with light Wilson fermions. In the final section, we shall use this model to perform a series of numerical tests on a recently proposed cure of the exceptional configuration problem [1] which suggests that greatly improved results are indeed possible for calculations using Wilson-Dirac fermions in the low mass chiral regime.

Following the example of Smit and Vink [5], we can take advantage of the fact that 2-dimensional QED (massive Schwinger model) provides a particularly useful testbed for investigating the pathologies of the quenched approximation. As in 4-dimensional QCD, there is an intimate connection between the topological structure of the theory and the spectrum of the Dirac operator near the origin, which controls the chiral limit behavior. Moreover, proposed modifications of the quenched approximation can be tested against the full theory (including determinant effects) in great detail in the 2-dimensional case.

On large lattices at weak coupling the Wilson spectrum $\left\{\lambda_{a}\right\}$ settles (taking QED2 as an example) near the real axis into four distinct branches at $\operatorname{Re}(\lambda)= \pm 2,0$, with the central region containing two branches. It is usual to select the undoubled left branch (see Fig(1)) as a model for the continuum theory by tuning the bare quark parameter $m$ appropriately. For example, in QED2 one finds [5] a critical line at $\operatorname{Re}(\lambda) \approx-2+0.65 / \beta$, with a dispersion of the real part of the eigenvalue around this value which grows rapidly as one goes to stronger coupling. In particular, at stronger coupling one finds an increasing density of exactly real eigenmodes straying to the left away from the critical line into the region corresponding to small positive quark masses. Consequently, evaluation of the propagator in configurations giving 
rise to such modes necessarily results in noisy results, as one occasionally ends up sitting very close to a pole of the propagator. In a sense, the original rationale for moving from Minkowski to Euclidean space - avoiding the poles of the propagator at Minkowski momenta- has been vitiated by the complex (i.e. non-skewhermitian) character of the Wilson-Dirac operator. The exceptional configurations observed in quenched calculations appear to be due to precisely this feature of the Wilson action, and the study of these configurations reduces essentially to a study of the frequency and location of the exactly real eigenvalues of the Wilson-Dirac matrix defining the quadratic fermion action of a lattice theory.

The present paper is organized as follows. In Section 2 we review some of the basic properties of the Wilson-Dirac matrix in lattice gauge theory. Most of the results listed here are well known but the review allows us to clarify our notation and to remind the reader of some exact properties of the Wilson-Dirac spectrum following purely from linear-algebraic considerations. Results are given here both for QED in 2 and QCD in 4 dimensions. Section 3 contains a more detailed analysis of the mass singularity structure of the quenched functional integral. It is shown that the quenched functional integral as defined in a Monte Carlo simulation is strictly speaking undefined. The functional integral must be defined by analytic continuation from a pole-free region of the complex quark mass plane. In the region of physical interest, the path integral defines a cut function in this plane with a complicated branch structure. Section 4 describes the relationship between topological charge and the exactly real modes responsible for the pathologies of the quenched approximation. In Section 5 we study the dependence of the real part of the Wilson-Dirac spectrum on lattice volume and lattice spacing. In Section 6 we give the results of a detailed study of the changes in the real spectrum induced by the addition of a clover improvement term [6] to the action. Finally, in Section 7 we test the validity of a recently proposed [4] 
modified quenched approximation (MQA) which resolves the singularities introduced by real eigenmodes by a simple pole-shifting procedure. In particular, we carry out an explicit comparison of the MQA with both the naive quenched and full dynamical results for correlators of quark bilinears.

\section{Formal properties of the Wilson-Dirac operator}

The Euclidean Dirac operator $\not D(A)$ in the continuum is formally skew-hermitian for smooth gauge fields $A$, so at a formal level the quark propagator $(\not D(A)+m)^{-1}$ exists for all nonzero quark mass $m$. The existence of the inverse at zero quark mass is related to the topological structure of the background gauge field by the Atiyah-Singer index theorem. For example, in 2D QED, defining the Dirac operator on a compact 2-sphere of radius $R$ by the usual one-point compactification [7] gives a rigorously self-adjoint $i \not D(A)$ (for gauge fields which approach pure gauge at Euclidean infinity) with a purely discrete spectrum :

$$
\begin{aligned}
i \not D(A) \chi_{a}(x) & =\lambda_{a} \chi_{a}(x) \\
\int d^{2} x \frac{2 R}{x^{2}+R^{2}} \chi_{a}^{\dagger}(x) \chi_{b}(x) & =\delta_{a b} \\
\frac{e}{4 \pi} \epsilon_{\mu \nu} F_{\mu \nu}+\frac{2 R}{x^{2}+R^{2}} \sum_{a} \chi_{a}^{\dagger} \gamma_{5} \chi_{a}(x) & =\text { pure divergence }
\end{aligned}
$$

The eigenfunctions $\chi_{i}$ are normalizable relative to the inner product (2), and the "pure divergence" referred to in (3) is just the divergence of the axial vector current of the model, which is seen to reduce to a sum of a topological term and a sum over the $\gamma_{5}$ eigenvalues of each of the discrete modes. The latter sum (as a consequence of $\left.\left\{\gamma_{5}, \not \supset(A)\right\}=0\right)$ reduces to a sum over zero modes only, and therefore to a difference of the number of positive and negative chirality zero mode solutions of the Dirac equation in the background field. Nielsen and Schroer have given an explicit analytic expression for these solutions in an arbitrary smooth (and asymptotically pure-gauge) 
field. Essentially the same statements hold for QCD in 4 dimensions, with the obvious replacement of $\epsilon_{\mu \nu} F_{\mu \nu}$ by the appropriate 4-dimensional Chern-Simons $F \tilde{F}$ term.

Once the Euclidean space-time is discretized as in lattice theory, little of the preceding formalism remains in exact form, though qualitative remnants of the zero mode structure can be observed provided one works on large lattices with very smooth background gauge fields. In the first place, although a discrete spectrum is now guaranteed (the Dirac operator is a finite matrix!), the addition of a Wilson term to eliminate doubling destroys the skew-hermitian nature of the Dirac operator. Specifically, quark propagators are inverses of a matrix $D-r W+m \equiv \mathcal{M}+m$, with $D, W$ and $m$ the naive Dirac matrix, $W$ the Wilson term, and $m$ a quark mass parameter:

$$
\begin{aligned}
\mathcal{M} & \equiv D-r W \\
D_{a \alpha \vec{m}, b \beta \vec{n}} & =\frac{1}{2}\left(\gamma_{\mu}\right)_{a b} U_{\alpha \beta}(\vec{m} \mu) \delta_{\vec{n}, \vec{m}+\hat{\mu}}-\frac{1}{2}\left(\gamma_{\mu}\right)_{a b} U_{\alpha \beta}^{\dagger}(\vec{n} \mu) \delta_{\vec{n}, \vec{m}-\hat{\mu}} \\
W_{a \alpha \vec{m}, b \beta \vec{n}} & =\frac{1}{2} \delta_{a b}\left(U_{\alpha \beta}(\vec{m} \mu) \delta_{\vec{n}, \vec{m}+\hat{\mu}}+U_{\alpha \beta}^{\dagger}(\vec{n} \mu) \delta_{\vec{n}, \vec{m}-\hat{\mu}}\right)
\end{aligned}
$$

Here $a, b$ are Dirac indices, $\alpha, \beta$ color indices, $\vec{m}, \vec{n}$ lattice sites, and $r$ the Wilson parameter, which must be chosen $0<r \leq 1$ and is usually taken to be unity. As $D$ is skew-hermitian and $W$ hermitian, the full Wilson-Dirac $\mathcal{M}=D-r W$ matrix is complex, and the generic eigenvalue (which locates poles of the quark propagator in the complex mass plane) will also be complex.

It will frequently be convenient to use a single index $i \equiv(a \alpha \vec{m})$ to identify the single Grassmann variable for a quark field component, and to write the WilsonDirac matrix elements with the notation $\mathcal{M}_{i j}$. From (6) it follows immediately that although $\mathcal{M}$ is neither skewhermitian (unless $r=0$ ) nor hermitian, the matrix $\gamma_{5} \mathcal{M}$ is hermitian. Consequently the coefficients of the secular equation for $\mathcal{M}$ are real:

$$
\operatorname{det}\left(\lambda-\gamma_{5} \mathcal{M}\right)=\left(\operatorname{det}\left(\lambda^{*}-\gamma_{5} \mathcal{M}\right)\right)^{*}
$$




$$
\operatorname{det}(\lambda-\mathcal{M})=\left(\operatorname{det}\left(\lambda^{*}-\mathcal{M}\right)\right)^{*}
$$

so that if $\lambda$ is an eigenvalue of $\mathcal{M}$, so is $\lambda^{*}$ - the eigenvalues are therefore either real or appear in conjugate pairs. The nearest neighbour structure of $\mathcal{M}$ also implies immediately that

$$
\operatorname{Tr}\left(\mathcal{M}^{n}\right)=0, \quad n \text { odd }
$$

which implies that odd powers of $\lambda$ are absent in the secular equation $\operatorname{det}(\lambda-\mathcal{M})=0$. Consequently, if $\lambda$ is an eigenvalue, so is $-\lambda$. The generic case therefore has the roots of the secular equation for $\mathcal{M}$ appearing as quartets $\alpha,-\alpha, \alpha^{*},-\alpha^{*}$ or as pairs of real eigenvalues $r,-r$. To summarize, we may write (the dimension $N$ is $12 V$ for $4 \mathrm{D}$ QCD, $2 V$ for $2 \mathrm{D}$ QED, where $V$ is the lattice volume = number of lattice sites)

$$
\operatorname{det}(\lambda-\mathcal{M})=\lambda^{N}+c_{2} \lambda^{N-2}+c_{4} \lambda^{N-4}+\ldots c_{N}
$$

where the $c_{i}$ are all real and given in terms of $\mathcal{M}$ by standard trace formulas

$$
\begin{aligned}
c_{2} & =-\frac{1}{2} \operatorname{Tr}\left(\mathcal{M}^{2}\right) \\
c_{4} & =-\frac{1}{4}\left(\operatorname{Tr}\left(\mathcal{M}^{4}\right)-\frac{1}{2} \operatorname{Tr}\left(\mathcal{M}^{2}\right)^{2}\right) \\
c_{6} & =-\frac{1}{6}\left(\operatorname{Tr}\left(\mathcal{M}^{6}\right)+\frac{1}{8} \operatorname{Tr}\left(\mathcal{M}^{2}\right)^{3}-\frac{3}{4} \operatorname{Tr}\left(\mathcal{M}^{2}\right) \operatorname{Tr}\left(\mathcal{M}^{4}\right)\right), \quad \text { etc }
\end{aligned}
$$

Henceforth we suppose that $r=1$. The factors $1 \pm \gamma_{\mu}$ appearing in $\mathcal{M}$ are then projection operators, and in particular $\left(1+\gamma_{\mu}\right)\left(1-\gamma_{\mu}\right)$ vanishes. Consequently, all terms in $\operatorname{Tr}\left(\mathcal{M}^{2}\right)$, which necessarily correspond to products $\mathcal{M}_{i j} \mathcal{M}_{j i}$ where $i, j$ involve nearest neighbour lattice sites, automatically vanish. As a result

$$
c_{2}=\operatorname{Tr}\left(\mathcal{M}^{2}\right)=0
$$

and the sum of squares of the complex eigenvalues of the Wilson-Dirac matrix must vanish in an arbitrary gauge configuration! This sum rule is very useful in checking 
the accuracy of the (sometimes unstable) numerical routines we have used to effect the spectral resolution of $\mathcal{M}$. The calculation of $c_{4}$ is equally straightforward. Again, each contributing term in $\operatorname{Tr}\left(\mathcal{M}^{4}\right)=\mathcal{M}_{i j} \mathcal{M}_{j k} \mathcal{M}_{k l} \mathcal{M}_{l i}$ corrresponds to a sequence of 4 links forming a closed path and with (for $r=1$ ) backtracks forbidden. The resulting color trace simply produces the plaquette trace for an elementary square on the lattice, provided that the linear dimension of the lattice (in all directions) is greater than 4 , so that Polyakov lines stretching across the whole lattice are excluded. In $\mathrm{D}=2,4$ dimensions, one finds

$$
\begin{aligned}
\operatorname{Tr}\left(\mathcal{M}^{4}\right) & =-D \sum_{P} \operatorname{Re} \operatorname{Tr}\left(U_{P}\right) \\
c_{4} & =\frac{D}{4} \sum_{P} \operatorname{Re} \operatorname{Tr}\left(U_{P}\right)
\end{aligned}
$$

which gives the sum rule that the fourth power of eigenvalues of $\mathcal{M}$ must sum to the gauge action. Similarly, $c_{6}, c_{8}, .$. involve sums of non-backtracking gauge-invariant loops (and eventually, Polyakov lines) of length 6,8 , etc. Note the symmetry property under simultaneous inversion of all links (which will be used below in discussing the analytic structure of quenched amplitudes $) c_{n}\left(\left\{U_{l}\right\}\right)=c_{n}\left(\left\{U_{l}^{-1}\right\}\right)$.

Similar calculations show that the quantity $\operatorname{Tr}\left(\mathcal{M M}^{\dagger}\right)$ is independent of the gauge field configuration. Namely

$$
\begin{aligned}
& \operatorname{Tr}\left(\mathcal{M M}^{\dagger}\right)=4 V \quad(2 \mathrm{D} \quad \mathrm{QED}) \\
& \operatorname{Tr}\left(\mathcal{M M}^{\dagger}\right)=16 N_{c} V \quad(4 \mathrm{D} \quad \mathrm{QCD})
\end{aligned}
$$

This implies, for example, that the spectrum of $\mathcal{M}$ is rigorously contained within a circle of radius $2 L$ for 2 dimensional lattice QED on a $L x L$ lattice. A stronger bound follows from the observation that (for $r=1$ ) $\operatorname{Tr} \mathcal{M}^{n}<C \cdot 3^{n}$ for large $n$ as the number of closed non-backtracking paths of length $n$ is clearly $<3^{n}$ in 2 dimensions and the Dirac traces involve products of projection operators which do not grow with $n$. This 


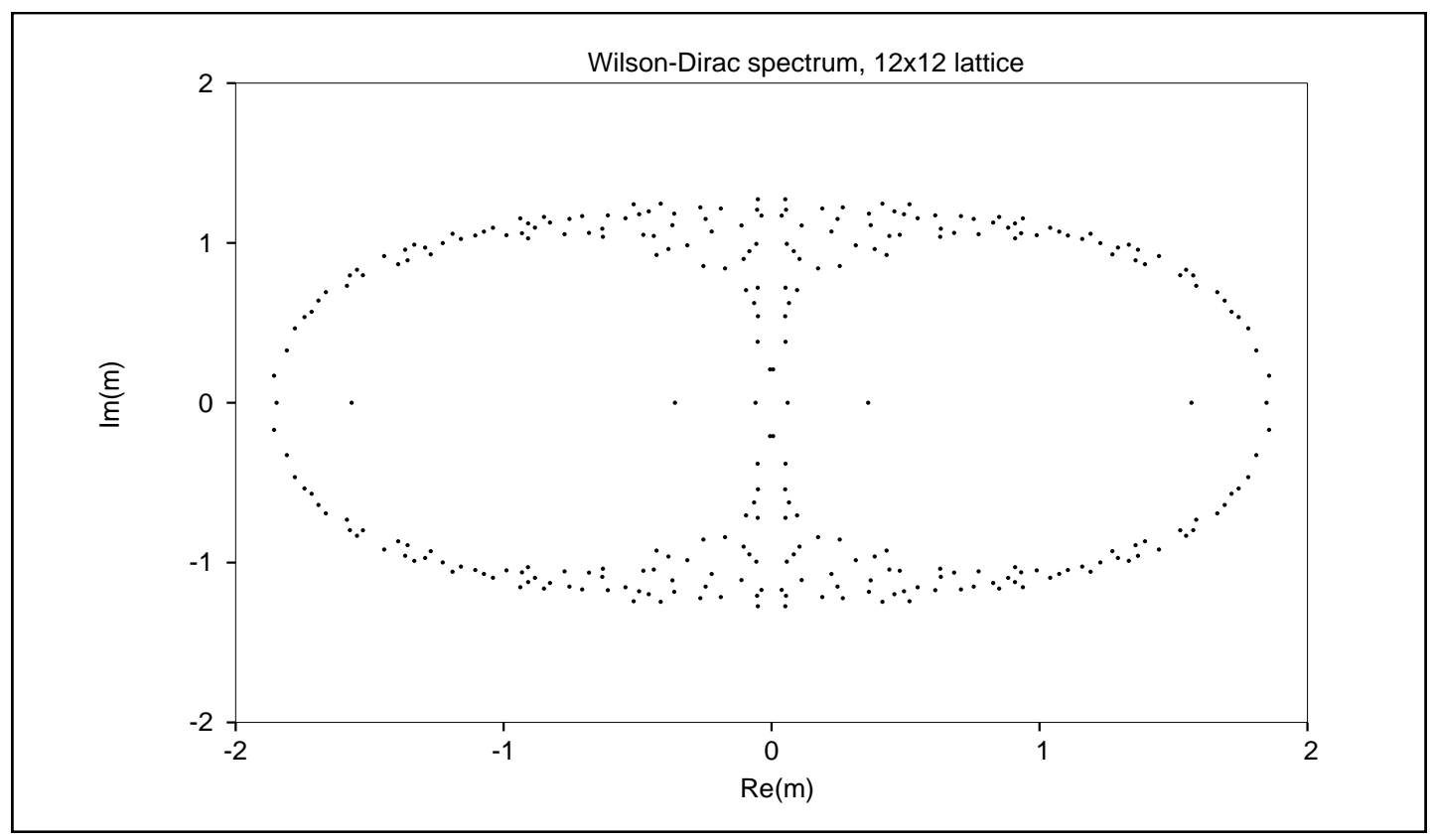

Figure 1: A typical Wilson-Dirac spectrum in QED2

implies $\left|\lambda_{a}\right|<3$ for eigenvalues of $\mathcal{M}$. In fact with typical gauge field configurations, eigenvalues are not found with magnitude larger than 2. A typical spectrum for quenched 2D QED (12x12 lattice at $\beta=4)$ is shown in Fig(1). The left, right and central branches of the Wilson-Dirac spectrum are clearly visible, as well as a pair of exactly real eigenvalues for each branch. One of these real modes has drifted quite a substantial distance into the interior of the spectral oval, away from the critical regime.

The discrete symmetries of the Wilson matrix mean that the spectrum is considerably more constrained than would be the case for a generic complex operator. In particular the appearance of exactly real eigenvalues as in Fig(1) is not a miracle. As a simple toy example, consider $N=4$, and assume that the secular equation is constrained, as for our Wilson matrix, to have only even terms with real coefficients. For a general value of the $r$ parameter (not equal to unity), we would have $c_{2} \neq 0$. The resulting 4 roots are all real provided only that $c_{2}<0, c_{2}^{2}>c_{2}^{2}-4 c_{4}>0$. The 
reality of such roots is clearly robust with respect to small variations of $c_{2}, c_{4}$. (For $r=1$, similar arguments show the robustness of the reality of roots of the cubic secular equation for $N=6$ ). Explicit calculations (described below) of the full spectrum in 2D lattice $\mathrm{QED}$ on a variety of lattices confirm that when real eigenvalues of $\mathcal{M}$ appear for some set of link angles, they remain real in a finite neighborhood of this point in the configuration space of gauge link variables. The appearance of exactly real modes may seem unsurprising in view of the necessity of such modes in a topologically nontrivial background in the continuum, but such modes do not appear in the Kogut-Susskind (KS) formulation of staggered fermions on the lattice [8], where the fermion matrix (essentially $D$ of $(5)$ ) is exactly skew-hermitian, so that the spectrum is confined to the imaginary axis, and an exactly zero eigenvalue is nongeneric. Instead, the latticized KS matrix displays pure imaginary eigenvalues close to, but not exactly, zero in a smooth background lattice field (e.g. after cooling) of nonzero winding number.

\section{Mass Singularity Structure of the Quenched Func- tional Integral}

The appearance of poles in the physical region for bare quark masses is a symptom of a serious disease in the standard quenched functional integral. Strictly speaking, this integral - even on a finite lattice, with compact integrations over gauge link variables- does not exist for values of the bare quark mass $m$ in the physical range. The quenched functional integral can in principle be defined by analytic continuation from a pole-free region (see below) in the complex plane of $m$, but as we shall soon show the analytic structure of quenched amplitudes becomes extremely complicated in the region of physical interest. In any event analytic continuation is out of the question for a numerically computed amplitude. We are forced to face the unpleasant 
fact that the quenched functional integral with Wilson fermions, as defined in a manner amenable to Monte Carlo sampling, runs directly over poles of the integrand. Staying at large quark masses would avoid this problem, which only appears, as we shall see, at kappa values $\kappa>\kappa_{0}$, where $\kappa_{0}$ is the critical kappa value for the free theory ( $=0.25$ in 2 dimensions, 0.125 in 4 dimensions). On the other hand, Monte Carlo simulation of the quenched integral at smaller, physically interesting bare quark mass will necessarily give ill-defined results, even when very large ensembles are used.

In a full dynamical simulation of hadron correlators in lattice QCD, one computes the functional integral over both fermionic and gauge degrees of freedom of a product of an even number of quark fields

$$
\begin{aligned}
&<\psi_{i_{1}} \psi_{i_{2}} . . \psi_{i_{n}} \overline{\psi_{j_{1}}} \overline{\psi_{j_{2}} . . \bar{\psi}_{j_{n}}>}= \frac{1}{Z_{0}(m)} \int d \psi_{i} d \overline{\psi_{j}} d U_{l} \psi_{i_{1}} \psi_{i_{2}} . . \psi_{i_{n}} \overline{\psi_{j_{1}}} \overline{\psi_{j_{2}} . . \overline{\psi_{j_{n}}}} \\
& e^{-S_{g}\left(U_{l}\right)-\overline{\psi_{i}}\left(m+\mathcal{M}_{i j}\right) \psi_{j}} \\
& \equiv \frac{N(m)}{Z_{0}(m)}
\end{aligned}
$$

Such 2n-point functions are necessarily meromorphic functions of the bare quark mass provided the determinant arising from the Grassmann integration is properly included. Indeed, by the usual rules of Grassmann integration, on a finite lattice both the numerator $N(m)$ and the vacuum amplitude $Z_{0}(m)$ in (13) are polynomials in the quark mass. In the quenched approximation, on the other hand, the absence of the determinantal factor results in a much more complicated analytic structure in the complex $m$-plane, as poles in quark propagators $D_{i j} \equiv(\mathcal{M}+m)_{i j}^{-1}$ remain uncancelled by the corresponding zeroes in $\operatorname{det}(\mathcal{M}+m)$. The position of these poles in the complex $m$-plane is of course dependent on the gauge field and consequently the location of singularities in $m$ of $N(m)$, where the gauge field integration has been performed, depends on whether the gauge field integrals are prevented from deformation by either a pinch or an end point singularity. (Of course, in the quenched 
approximation, the normalizing denominator $Z_{0}(m)$ only involves the pure gauge action and is consequently independent of $m$, so the entire singularity structure derives from the numerator in (13).) The generic structure of a quenched contribution to $N(m)$ is thus

$$
\int \Pi_{l} d U_{l} \frac{1}{\left(m^{N}+c_{2}\left(U_{l}\right) m^{N-2}+c_{4}\left(U_{l}\right) m^{N-4}+. . c_{N}\left(U_{l}\right)\right)^{q}} \cdot\left(\text { analytic in } \mathrm{m}, \mathrm{U}_{1}\right. \text { ) }
$$

where $q$ is the number of quark propagators appearing in the correlator. Here we are examining the analytic structure in the complex plane of a single quark massthe issue of several flavors, and the effect of hairpin graphs in isosinglet sectors in reducing the level of singularity in (21) will be discussed below. As the coefficients $c_{m}\left(U_{l}\right)$ are all gauge-invariant, a maximal tree of links may be assumed fixed to unity and the integrals in (21) only include physical degrees of freedom.

For the rest of this section we shall consider abelian gauge theory on a 2 dimensional $L \mathrm{x} L$ lattice, although much of the discussion applies just as well to 4D QCD. The gauge integrations are then over link angles $0 \leq \theta_{l} \leq 2 \pi, 1 \leq p$ where $p$ is the number of physical (non-fixed) links. From the bound on the magnitude of eigenvalues of $\mathcal{M}$ implied by (17), it is clear that $N(m)$ is analytic for $|m|>2 L$, and that singularities are confined to a compact central region around the origin of the $m$ plane with poles (if any) and paired branch points arranged symmetrically around the origin and real axis. Alternatively, defining $z_{l} \equiv e^{i \theta_{l}}$, the link integrations can be thought of as over a $p$-dimensional torus $T$, with singularities in $m$ arising whenever two or more root surfaces $m^{N}+c_{2}\left(z_{l}\right) m^{N-2}+\ldots c_{N}\left(z_{l}\right)=0$ pinch $T$ from opposite sides. That branch point singularities can easily arise in this fashion is readily seen from a simple example (involving only a single physical link) where the integral over the link variable $z \equiv e^{i \theta}$ is written as a complex integral over the unit circle

$$
f(m) \equiv \oint \frac{d z}{2 \pi i z} \frac{1}{m^{4}+\alpha(z+1 / z) m^{2}+1}=\frac{1}{\sqrt{\left(1+m^{4}\right)^{2}-4 \alpha^{2} m^{4}}}
$$


Here $\alpha$ is a real constant. The branch point singularities located at the roots of $\left(1+m^{4}\right)^{2}=4 \alpha^{2} m^{4}$ arise from a pinch of the unit circle integration contour by the two roots in $z$ of the integrand in (22). More generally, the roots of $\operatorname{det}\left(m-\mathcal{M}\left(z_{l}\right)\right)$ define singularity surfaces of the integral

$$
\begin{aligned}
f(m) & =\oint \frac{1}{\operatorname{det}\left(m-\mathcal{M}\left(z_{l}\right)\right)} \prod_{l} \frac{d z_{l}}{2 \pi i z_{l}} \\
& =\oint \frac{1}{m^{N}+c_{2}\left(z_{l}\right) m^{N-2}+\ldots} \prod_{l} \frac{d z_{l}}{2 \pi i z_{l}}
\end{aligned}
$$

This integral is well-defined for $|m|>2 L$, and defines a real-analytic function of $m$ there, but will encounter difficulties as $m$ is brought into the central region where roots of $\operatorname{det}\left(m-\mathcal{M}\left(z_{l}\right)\right)$ can occur. In particular, branch points of $f(m)$ in the complex plane of the mass variable $m$ will occur at points $m_{0}$ whenever [9] the hypertorus of integration in (23) is pinched from opposite sides by two branches of the singularity surface $S_{1}\left(z_{l}, m_{0}\right)$ and $S_{2}\left(z_{l}, m_{0}\right)$ with

$$
\alpha_{1} \frac{\partial S_{1}}{\partial z_{l}}+\alpha_{2} \frac{\partial S_{2}}{\partial z_{l}}=0
$$

for fixed $\alpha_{1}, \alpha_{2}$ and all links $z_{l}$. For example, setting all $z_{l}=1$ (the ordered free configuration), there is (for periodic boundary conditions) always a pair of real roots at $m_{0}= \pm 2$. If $m_{0}$ is moved infinitesimally away from these points (say to $m_{0}=2 \pm \epsilon$ ) roots of $\operatorname{det}\left(m-\mathcal{M}\left(z_{l}\right)\right)$ appear with $\left|z_{l}\right|<1$ and $\left|z_{l}\right|>1$, where the configuration $\left\{z_{l}^{-1}\right\}$ is necessarily a root if $\left\{z_{l}\right\}$ is (as $c_{n}\left(z_{l}\right)=c_{n}\left(z_{l}^{-1}\right)$ ). Thus the singularity surfaces are naturally paired by taking $S_{2}\left(z_{l}, m\right) \equiv S_{1}\left(z_{l}^{-1}, m\right)$, in which case (25) is automatically satisfied with $\alpha_{1}=\alpha_{2}$. The surfaces come together and pinch the integration hypertorus at a single point provided $z_{l}=z_{l}^{-1}$ for all links, i.e. if $z_{l}= \pm 1$. We conclude that branch points of $f(m)$ are present at all complex values of $m$ which solve $\operatorname{det}\left(m-\mathcal{M}\left(z_{l}\right)\right)=0$ for any configuration where the link variables are either 1 or -1 . In particular, the eigenvalues of $\mathcal{M}$ for the ordered configuration are all 


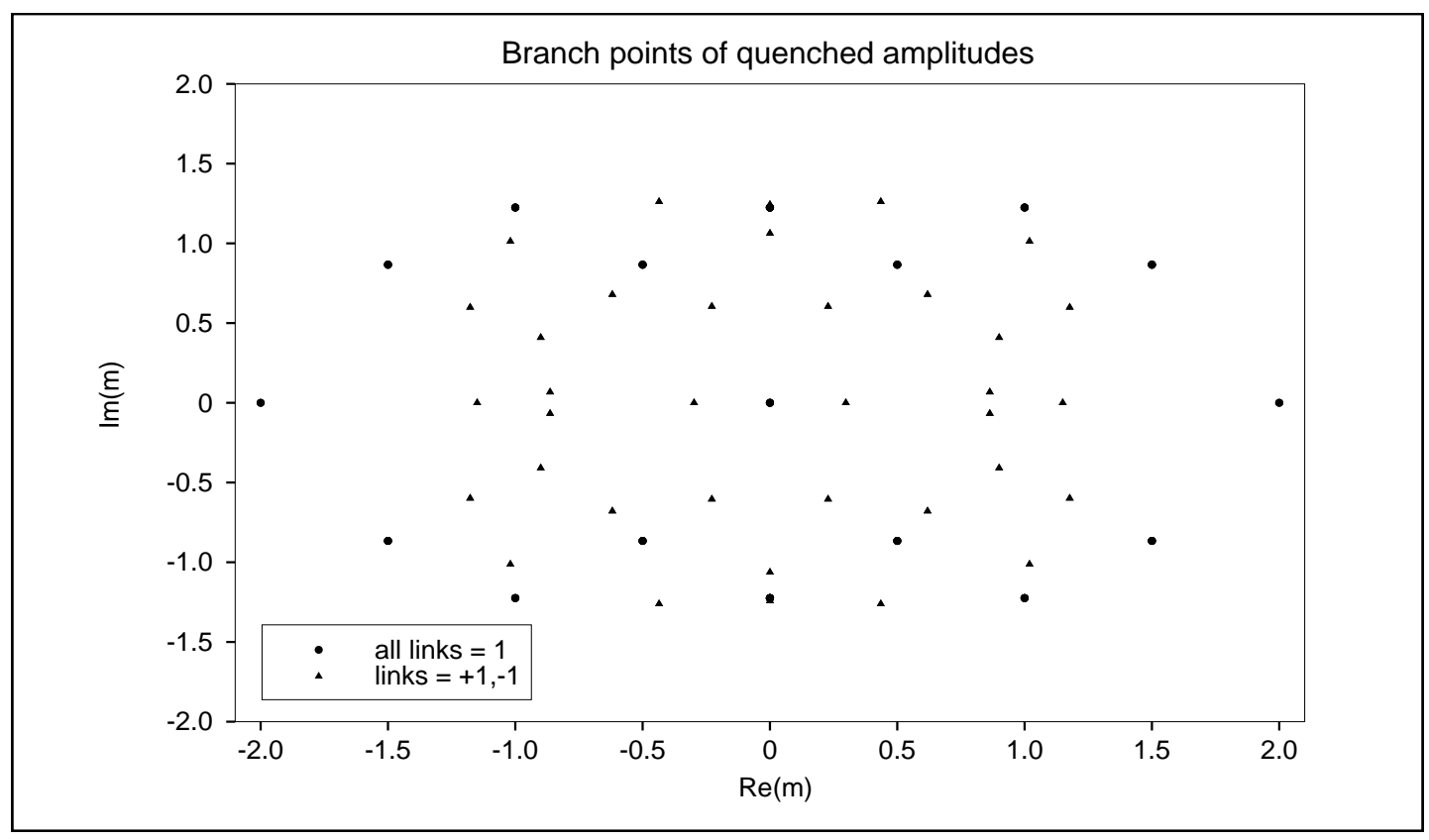

Figure 2: Location of some of the branch points for $\mathrm{L}=6$

branch points of the quenched amplitudes. In addition, there are many other branch points corresponding to configurations where the link variables are allowed to take the values \pm 1 . A glance at Fig(3), which shows branch points arising from the ordered configuration as well as from a disordered configuration where the link variables were set to \pm 1 randomly (on a $6 x 6$ lattice), indicates just how complicated the analytic structure of a lattice quenched amplitude can be. In particular, branch cuts extend along the entire real axis from $m=-2$ to $m=2$, exactly in the region where one attempts to approach continuum behavior. 


\section{Zero modes, topological charge, and cooling}

As discussed above in Section 2, there is a clear connection in the continuum theory between the topological charge of the background gauge configuration and the zero mode structure of the Dirac operator. On the lattice, this connection is necessarily less precise, as discretization effects smear out both the shape of the Dirac spectrum and alter the value of the topological charge associated with a given lattice gauge field. As discussed by Smit and Vink, two possible definitions of topological charge for 2D QED on the lattice are

$$
Q_{1} \equiv \frac{1}{2 \pi} \sum_{P} \sin \left(\theta_{P}\right)
$$

where $\theta_{P}$ is the plaquette angle for plaquette $P$. This definition of course gives in general a noninteger value for the topological charge. Alternatively, one may define

$$
Q_{2} \equiv \frac{1}{2 \pi} \sum_{P} \theta_{P}, \quad-\pi<\theta_{P} \leq \pi
$$

which gives an integer value for the topological charge, agreeing with (26) for smooth fields in the continuum limit. For a typical gauge configuration at weaker values of the coupling the number of exact zero modes found for the lattice Wilson-Dirac operator agrees with $Q_{2}$ (in each critical branch), with $Q_{2}$ essentially the closest integer to $Q_{1}$, while at lower $\beta$ one finds increasingly frequent discrepancies in these three measures of topological charge.

A convenient way of understanding the role of discretization on a given finite lattice involves cooling a Monte Carlo generated configuration to gradually remove highmomentum components from the gauge configuration. Such cooling tends to stabilize any nontrivial topology while bringing the zero mode and topological properties of the system closer to continuum-like behavior. A typical result of a series of cooling sweeps (in which the action is adiabatically lowered, effectively by running the simulation 
Table 1: Cooling a configuration with topological charge $=1$

\begin{tabular}{|c|c|c|c|c|c|}
\hline Cooling sweeps & $Q_{1}$ & $Q_{2}$ & Action & Real modes (left branch) & Chirality Fraction \\
\hline 0 & 0.850 & 1 & 20.8 & -1.892 & 0.930 \\
1 & 0.913 & 1 & 14.2 & -1.901 & 0.999 \\
2 & 0.940 & 1 & 10.1 & -1.911 & 0.999 \\
3 & 0.950 & 1 & 6.9 & -1.919 & 0.999 \\
4 & 0.959 & 1 & 4.7 & -1.926 & 0.999 \\
\hline
\end{tabular}

at a high fixed $\beta$ value) on the near-real-axis spectrum of $\mathcal{M}$ (left branch) is shown in Table(1). As the initial configuration, with topological charge $Q_{1}=0.85$ is cooled, the action decreases steadily, the topological charge $Q_{1}$ increases towards unity, and the norm fraction of the positive chirality part of the corresponding real eigenmode increases rapidly towards 1 . There is a single exactly real eigenmode (in each branch) of the Wilson-Dirac matrix throughout. Restricting our attention to the left critical branch only, cooling moves the real mode gradually further to the left (as we would expect by forcing the system to a larger effective $\beta$ ), but leaves the number of real modes unchanged. Note that the discrete definition $Q_{2}$ gives exactly unity for the topological charge throughout.

In the course of a quenched Monte Carlo simulation topological charge fluctuations arise resulting in the appearance of new exactly real eigenmodes of the Dirac operator. An interesting example of this phenomenon is illustrated in Fig.3. Initially (circular points) there is one exactly real eigenvalue per branch, and a pair of slightly off-realaxis complex modes considerably to the right of the left-branch critical line, which for the lattice in question $(12 \times 12$ at $\beta=5)$ is at $\operatorname{Re}(\mathrm{m}) \approx-1.91$. Both $Q_{1}$ and $Q_{2}$ give values for this initial Monte Carlo configuration close to $+1\left(Q_{2}\right.$ is of course exactly 1), as shown in Table(2). The corresponding eigenvector would have exactly positive chirality in the continuum- instead the norm fraction of the positive chirality piece is initially about $98.8 \%$ (Table(2), column 6). As the cooling proceeds, the complex pair 
Table 2: Effect of cooling on a topological charge fluctuation

\begin{tabular}{|c|c|c|c|c|c|}
\hline Cooling sweeps & $Q_{1}$ & $Q_{2}$ & Action & Real modes (left branch) & Chirality Fraction \\
\hline 0 & 1.167 & 1 & 42.4 & -1.90 & 0.988 \\
2 & 1.266 & 1 & 25.9 & -1.92 & 0.994 \\
4 & 1.486 & 2 & 15.7 & $-1.94,-1.71$ & $0.995,0.462$ \\
6 & 1.636 & 2 & 10.3 & $-1.95,-1.82$ & $0.995,0.673$ \\
8 & 1.693 & 2 & 7.45 & $-1.96,-1.86$ & $0.995,0.771$ \\
10 & 1.817 & 2 & 5.33 & $-1.96,-1.90$ & $0.997,0.897$ \\
\hline
\end{tabular}

in Fig(3) move onto the real axis (diamond points in Fig(3)) and then split laterally, with one member moving out towards the critical line. The topological charge $Q_{1}$ moves smoothly up towards 2 as this happens, with the discrete version $Q_{2}$ switching from 1 to 2 at roughly the point when the second exactly real mode appears (we take snapshots of the entire spectrum after each cooling sweep through the whole lattice, so it is difficult to be more precise as to the exact point at which the new real modes appear). Note that the "right chirality" fraction of the new zero mode is initially rather low (less than one-half), but increases steadily as this mode becomes stabilized and moves leftward into the critical region.

Evidently, in this case the initial configuration, with $Q_{1}, Q_{2}$ of order unity, actually contains a "nascent" topological charge 2 structure, with large high momentum fluctuations hiding the larger value of topological charge. The presence of this hidden topological charge is nevertheless signalled by the presence of complex pairs in the interior of the spectral oval, close to the critical region. In the case illustrated here, the effect of cooling mimics the further evolution under Monte Carlo updating, which also leads to $Q_{1} \approx 2$ after a few more sweeps, with a spectrum containing two exactly real modes per branch. In other cases, we have observed a reduction of topological charge under cooling: a pair of real eigenvalues in the left and central branches move out into the interior of the spectral oval, colliding there, then moving vertically away 


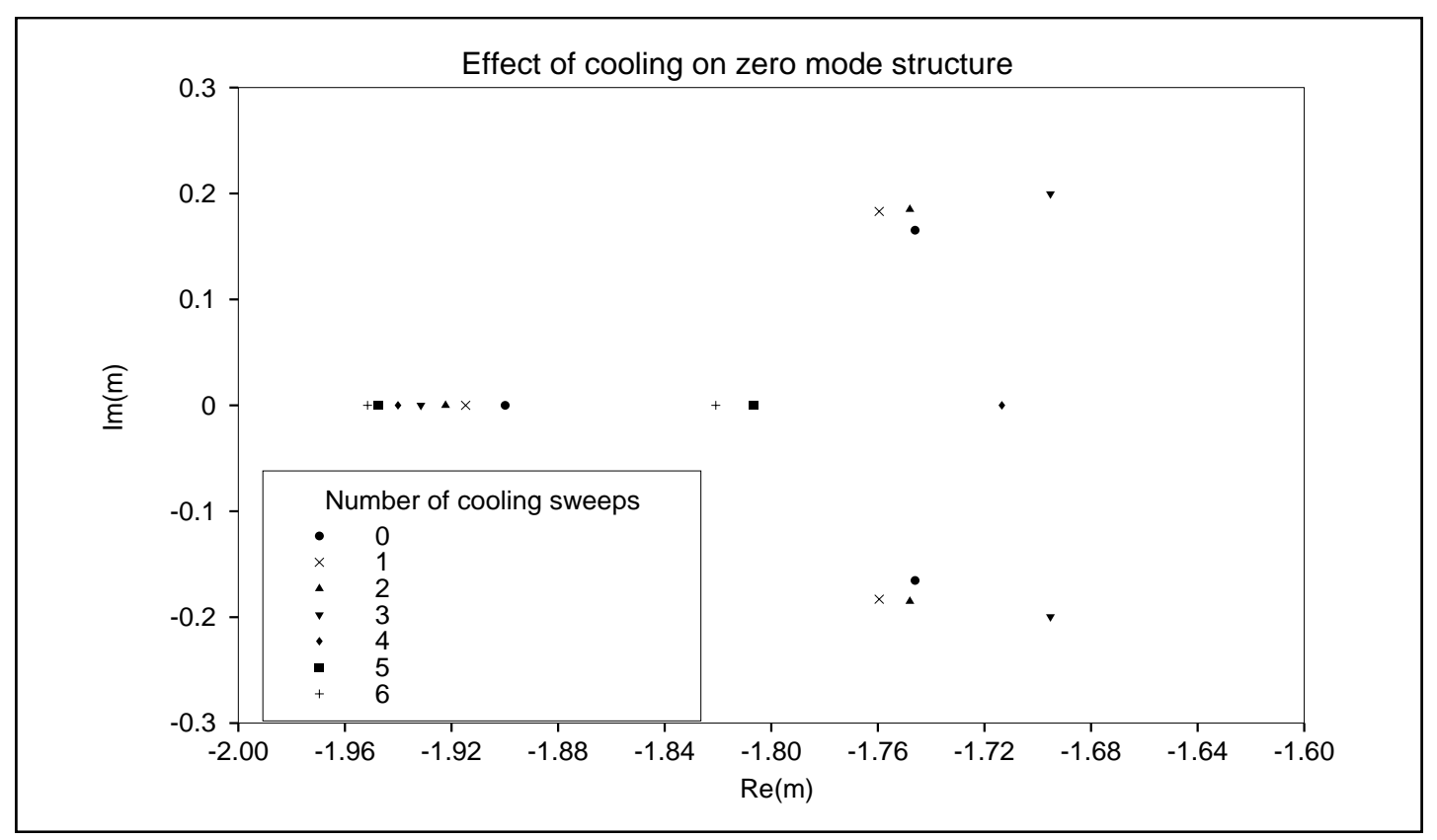

Figure 3: Effect of cooling on near-zeromodes $(\beta=5,12 \times 12$ lattice)

from the real axis, eventually to join the noncritical modes far from the real axis. 


\section{$5 \quad$ Lattice Volume and Lattice Spacing Dependence of Real Spectrum}

It has become part of the lore of quenched lattice QCD simulations that the exceptional configuration problem appears to be ameliorated at fixed $\beta$ (i.e. lattice spacing) when one goes to larger physical volumes. It is therefore of some interest to see whether this feature can be understood in terms of the volume dependence of the real part of the Wilson-Dirac spectrum. As one increases the physical volume, one naturally expects more frequent occurrence of configurations with nonvanishing topological charge, so it is clear that the overall number of exactly real zero modes, which as we have seen above are clearly correlated with nontrivial topological structures, should increase with volume. The appearance of exceptional configurations requires however that these modes appear in the quark mass regime being studied, just to the left of the critical line corresponding to zero renormalized mass.

The simulations described in the preceding section show that the number of exact zero modes is roughly correlated with the absolute magnitude of the topological charge $Q_{1} \equiv \frac{1}{2 \pi} \sum_{P} \sin \left(\theta_{P}\right)$. Quenched compact 2 dimensional QED is an exactly solvable model in the pure gauge sector, so it is a straightforward exercise to evaluate the distribution of this quantity over quenched configurations generated on a LxL lattice (periodic boundary conditions) at any given $\beta$. If the lattice volume is sufficiently

large that $\left(I_{1}(\beta) / I_{0}(\beta)\right)^{L^{2}}$ (which decreases exponentially fast with lattice volume) is negligible, where $I_{0}, I_{1}$ are the usual modified Bessel functions, then periodic and free boundary conditions become equivalent and the distribution of $Q_{1}$ becomes exactly Gaussian. (For the smallest lattice used below, $\mathrm{L}=6$ at $\beta=4$, this approximation incurs an error of order $10^{-3}$ ). One finds

$$
\rho\left(Q_{1}\right)=C e^{-2 \pi^{2} \frac{\beta I_{0}(\beta)}{I_{1}(\beta)} \frac{Q_{1}^{2}}{L^{2}}}
$$


which implies that the average value $<\left|Q_{1}\right|>$ rises linearly with the lattice size, or as the square root of the lattice volume. We have evaluated the spectra for 2500 configurations of lattices at $\beta=4$ and sizes $\mathrm{L}=6,8$, and 10 . The total number of exactly real modes rises in this range of lattice sizes somewhat faster than $\mathrm{L}$ though not as rapidly as $\mathrm{L}^{2}$ - specifically, the average number of exactly zero modes per branch was found to be $0.26,0.47$ and 0.63 for the $\mathrm{L}=6,8$, and 10 lattices respectively. The spectral histograms for these three cases in the region of the left critical branch is shown in Fig(4).

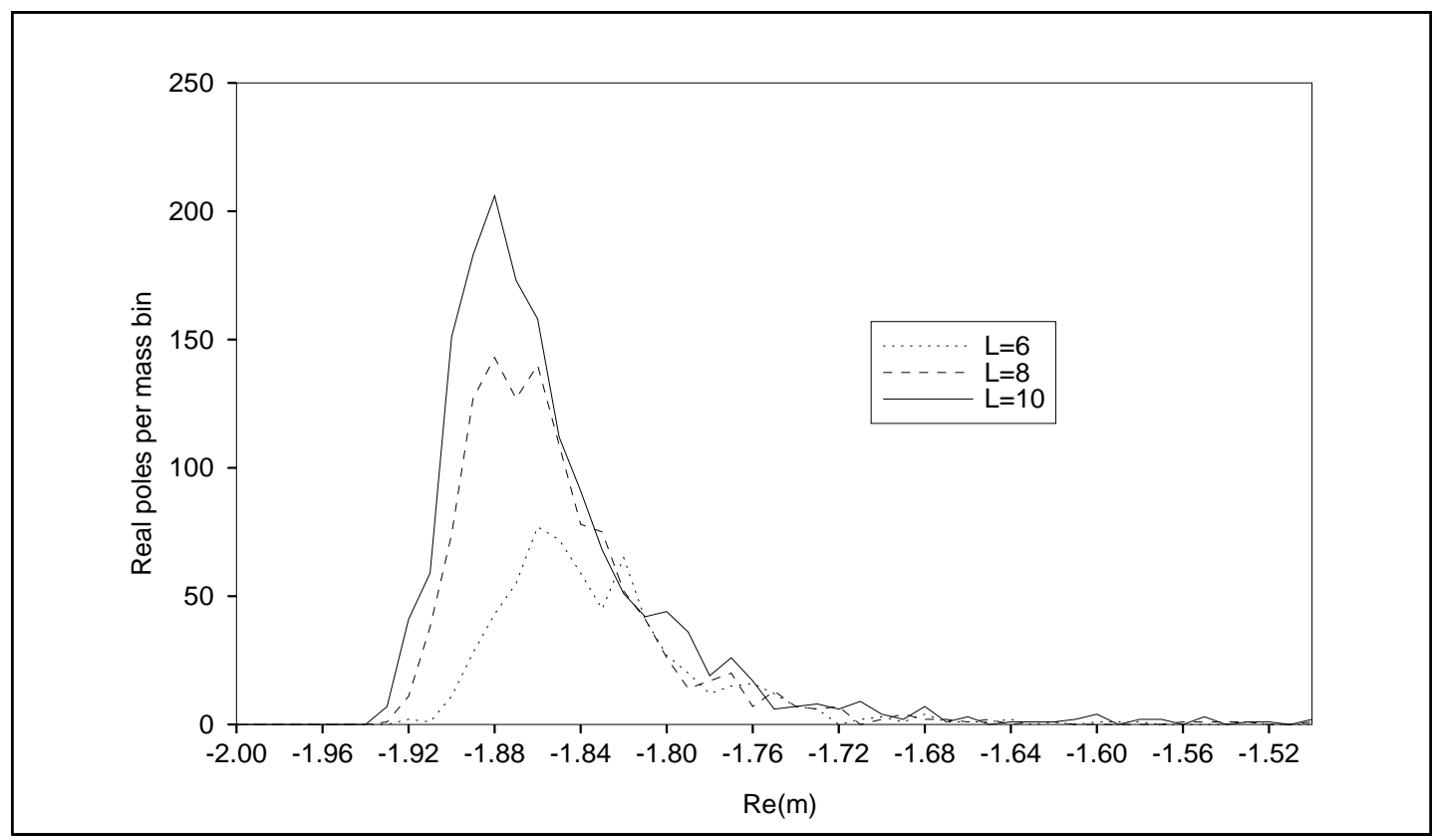

Figure 4: Volume dependence of spectrum (left branch))

Fig(4) provides a possible clue to the observation that exceptional configurations become rarer at fixed quark mass as the volume increases. We see that although the total number of real modes is certainly increasing with lattice volume, the leftmost zero mode shifts towards the boundary of the spectral region at $m=-2$ rather slowly (for $L=6,8$, and 10 , the leftmost modes were found at $-1.920,-1.923$, and -1.927 respectively), while the peak of the histogram, which we may identify with the critical mass 


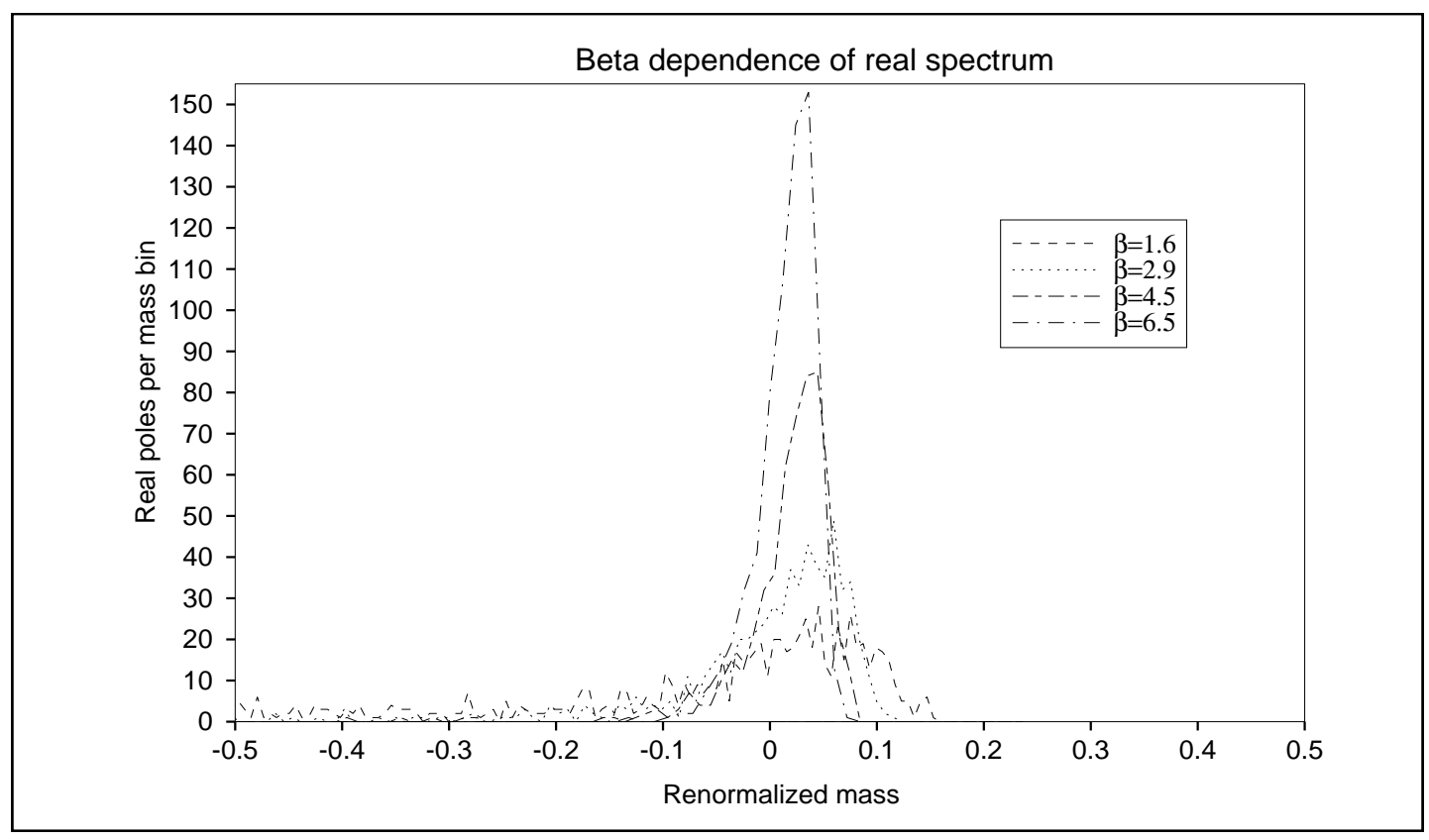

Figure 5: Beta dependence of spectral histogram

value, shifts leftward more rapidly (for $L=6,8$ and 10, the histograms peak at -1.855 , $-1.87,-1.88)$, so that the probability of encountering an exceptional configuration as one approaches the critical line from the left decreases with increasing volume if one keeps a fixed offset from the critical line to maintain a fixed physical quark mass. However, these histograms also make it clear that the exceptional configurations will necessarily appear at any volume once one goes sufficiently close to the critical point. As we shall now see, the benefits to be obtained from increasing lattice volume are far less dramatic than from the reduction of lattice spacing (at fixed physical volume).

In Fig(5) we show a histogram of exactly real eigenmodes appearing in quenched simulations in QED2 on $L \mathrm{x} L$ lattices with $L=6,8,10$ and 12 , this time with the gauge coupling readjusted to keep the physical lattice volume fixed (specifically, we have used $\beta=1.6,2.9,4.5$ and 6.5). The histograms were obtained from 1000 configurations separated by 200 quenched Monte Carlo sweeps (except for $L=12$, where measurements were separated by 500 sweeps). Note that the direction of the horizon- 
tal axis in these plots is reversed from the previous spectral plots, as by convention positive quark mass corresponds to the region to the left of the critical line (at roughly $2-0.65 / \beta)$. The density of real modes near the left critical branch is shown, relative to a critical line defined in this case by the peak of the distribution (this turns out to be close to but slightly offset from the Smit-Vink critical line value, with the offset decreasing with increasing $\beta$ ), and with the scale on the horizontal axis rescaled to keep physical masses fixed. It is apparent that the spread of dangerous poles into the physical mass region becomes acute at strong coupling, and that (at least in 2D QED) the probability of encountering exceptional configurations at a fixed renormalized quark mass decreases rapidly as $\beta$ is increased.

The manifest asymmetry of the real mode distribution around the critical line accounts for the uncontrollably noisy results obtained if one attempts a quenched simulation at lighter quark masses approaching from the interior of the spectral oval (i.e. at values of the kappa parameter greater than kappa critical). Nevertheless, we

shall see below that the modified quenched approximation approach 四 to resolving the exceptional configurations in the $\kappa<\kappa_{c}$ regime is equally successful in removing this noise and restoring sensible correlators for $\kappa>\kappa_{c}$. 


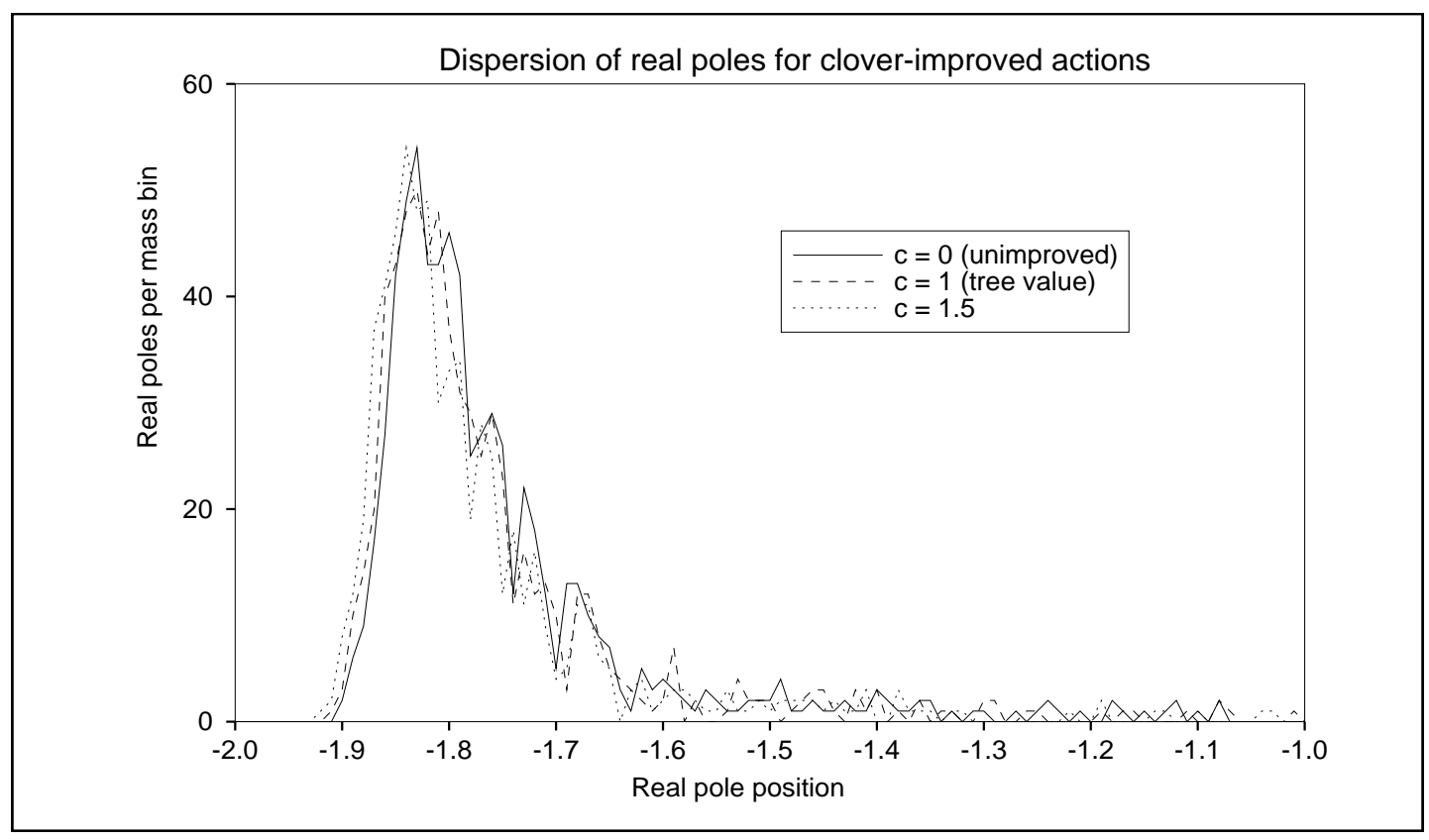

Figure 6: Dependence of spectral histogram on clover improvement coefficient $(\beta=4.5)$

\section{Effects of clover improvement on the Wilson- Dirac Spectrum}

The dispersion of real axis eigenvalues of the Wilson-Dirac matrix was seen above to decrease rapidly with increasing $\beta$, and it is clear that we are dealing with a pathology which is directly traceable to the particular discretization of the theory implied by an unimproved Wilson action for the quark fields. At first sight this would suggest that the problem might be removable (or at least, substantially reduced) by adopting a locally improved action, say by adding a clover-leaf term with appropriate coefficient. Unfortunately (see also [3]) this does not seem to be the case. In Fig.6 we show the effect of introducing such a term, with various values of the coefficient, on the spectral histogram of real eigenvalues for a $8 \times 8$ lattice at $\beta=4.5$ in QED2. Evidently, the clover improvement term is simply not effective in restraining the tendency of real modes to drift away from the critical region at strong coupling. 
Of course, the addition of a clover term certainly will shift the location of real modes on each individual configuration. Such a shift can have a dramatic effect on a given exceptional configuration, as it may be enough to move the propagator pole far enough from the chosen quark mass value to remove the large fluctuation in the correlators for that configuration. But in a statistical sense the problem remains. Our QED2 studies have revealed a rather interesting effect at the individual configuration level: it appears that the effect of a clover term is much stronger on the two central branches (not used in typical simulations) of the Wilson-Dirac spectrum. This effect is shown in $\operatorname{Fig}(7)$, where the full spectrum on a typical topological charge $Q_{2}=2$ configuration is shown for clover coefficient values of $0,1.2$, and 2.4 (where the tree value is unity). At higher values of the clover coefficient the real modes in the right central branch become degenerate and the nonzero modes line up quite accurately with the ones on the real axis. There is also a tendency for the two central branches to separate as the clover coefficient is made larger. The effect on the left and right lateral branches is less pronounced- the two zero modes on the left branch move somewhat apart, while those on the right move together. The whole process is reversed left to right if the sign of the clover coefficient is changed. 

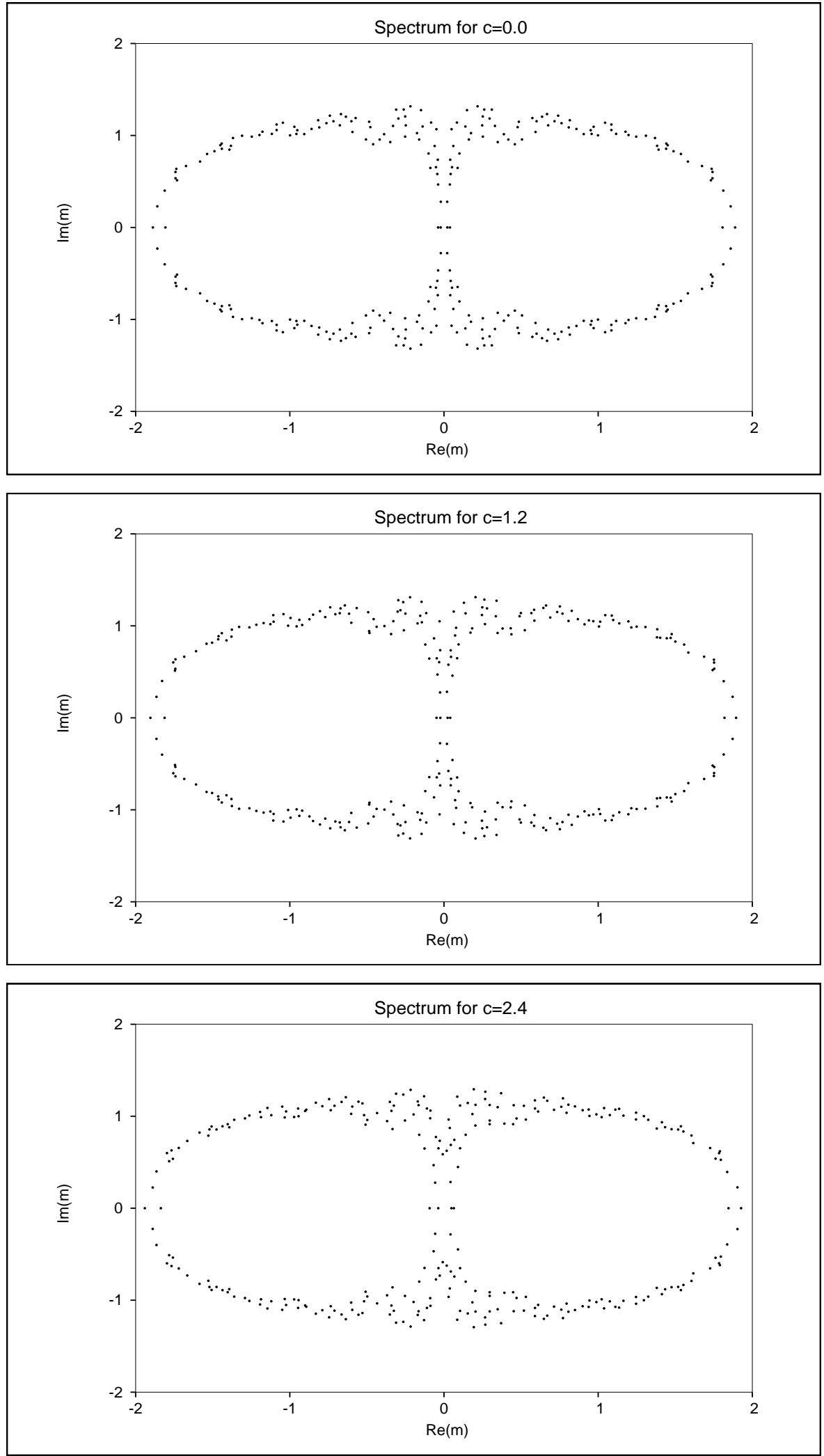

Figure 7: Spectrum of topological charge 2 configuration, clover coefficients $=0,1.2,2.4$ 


\section{Testing the modified quenched approximation}

Recently, we have proposed a nonlocal improvement procedure which should become arbitrarily precise in the continuum limit, as it involves explicitly shifting the position of real poles of the quark propagator by amounts which go to zero in this limit, but in such a way as to preserve the location of the critical point to leading order as the lattice spacing goes to zero. A redefinition of the Wilson-Dirac matrix which involves a shift in the location of real eigenvalues while leaving the corresponding eigenmodes unchanged evidently corresponds to a nonlocal addition to the action, as the typical eigenmodes for a real eigenvalue will be associated with a topological charge fluctuation which extends over several lattice sites (with power rather than

exponential falloff away from the "instanton center"). Of course, we need to be sure that this redefinition does not introduce an unwanted sizeable systematic distortion of the quenched averages at finite lattice spacing- in particular, does the proposed redefinition, which is really a prerequisite to having any precise notion of a quenched lattice amplitude, actually yield results which are less noisy and closer to the results of a full dynamical simulation on the same lattice? Direct evidence for this will now be presented.

The modified quenched approximation (MQA) recently proposed by us yields a well-defined regularization of the quenched functional integral which is constructed to reduce to the usual version in the continuum limit, in the sense that real axis poles of the Wilson-Dirac propagator which have strayed into the physical region for the quark masses are shifted back to the critical line where they would sit in this limit. The shift is performed in such a way as to preserve the location of the critical line in an average sense (for more details, see [四). In the context of QED2, we define the MQA modified propagator as follows. The fermion propagator $S \equiv\left(\frac{1}{2 \kappa}+\mathcal{M}\right)^{-\infty}$ 
(with the bare quark mass defined as $m_{0} \equiv \frac{1}{2 \kappa}-\frac{1}{2 \kappa_{c}}$ in terms of the usual kappa parameters, and with $\frac{1}{2 \kappa_{c}} \sim 2.0-0.65 / \beta \equiv \lambda_{c}$ in QED2) has the spectral decomposition

$$
S_{i j}=\sum_{a} \frac{\chi_{a i} \tilde{\chi}_{a j}}{m_{0}+\lambda_{c}+\lambda_{a}}
$$

where $\lambda_{a}$ are the eigenvalues of $\mathcal{M}$, and the indices $i, j$ incorporate spatial and Dirac information. The left-shifted real eigenvalues will cause poles at values of $m_{0}$ corresponding to $\kappa<\kappa_{c}$. For QED2 we are able to perform a full spectral resolution for each configuration . Define $u \equiv 2 m_{0}=\frac{1}{\kappa}-\frac{1}{\kappa_{c}}$ and replace any pole appearing at a position $u=u_{\text {pole }}$ as follows

$$
\frac{1}{u-u_{\text {pole }}} \rightarrow \frac{2}{u}-\frac{1}{u+u_{\text {pole }}}
$$

At large mass (or large $u$ ) the first two terms in the expansion in $1 / u$ are identical and terms linear in the shifts should average to zero. This procedure preserves the location of $\kappa_{c}$ to first order in the dispersion of real eigenmodes around the critical line (which is of course going to zero in the continuum limit). The full MQA propagator may be simply computed by adding a term to the naive fermion propagator $S_{i j}$ which incorporates the pole shift. Namely, for any configuration where a pole(s) appears in the physical region (operationally we have defined this in this paper to be the region between 0 and $2 m_{0}$ on the real bare quark mass axis), we replace

$$
S_{i j}^{\mathrm{MQA}} \equiv S_{i j}+A^{\mathrm{pole}}(\kappa) \operatorname{Res}_{i j}^{\mathrm{pole}}
$$

with

$$
\begin{aligned}
A^{\text {pole }}(\kappa) & \equiv \frac{2}{u}-\frac{1}{u+u_{\text {pole }}}-\frac{1}{u-u_{\text {pole }}} \\
\operatorname{Res}_{i j}^{\text {pole }} & \equiv \lim _{u \rightarrow u_{\text {pole }}}\left(u-u_{\text {pole }}\right) S_{i j}
\end{aligned}
$$

Although the procedure outlined here is certainly valid in the continuum limit, where we expect the frequency of exceptional configurations at any fixed physical 
quark mass to vanish, it is perhaps not apparent that the modification induced by the pole shift procedure does not introduce some large and misleading distortion of the correlators at stronger coupling. The level of numerical control possible with QED2 allows a direct comparison of the naive quenched, shifted pole, and full dynamical results for the interesting correlators of the theory. We have measured the pseudoscalar correlator $<\bar{\psi}(x) \gamma_{5} \psi(x) \bar{\psi}(0) \gamma_{5} \psi(0)>$ at fairly strong coupling $\beta=4.5$ on a 10x10 lattice for these three situations for a variety of quark masses close to the critical point of the left critical branch. The full dynamical simulations were done using an exact update algorithm [10] where the determinant and fermion propagator are known exactly at every stage. Typically, measurements were performed after every sweep for 400 sweeps, and the statistical error computed by measuring an autocorrelation time for each correlator (the naive quenched correlators decorrelate very rapidly so autocorrelation times are on the order of 1 sweep in the unshifted quenched case, while for the shifted pole and dynamical correlators, the autocorrelation times range from 3-10 sweeps). QED2 is a superrenormalizable theory with a finite bare coupling which fixes the mass scale of the theory, so a direct comparison of quenched and dynamical results can be made in this theory without the need for a compensating scale change. As we work on fairly small lattices, it is difficult to find mass plateaus suitable for extracting a meson mass, and we have decided to compare directly the pseudoscalar correlators, which are after all physical quantities with unambiguous values (for given $\beta$ and lattice size) in the full unquenched theory.

The comparison of naive quenched, MQA and full dynamical simulations for quark mass values $0.08,0.10$ and 0.115 are shown in $\operatorname{Fig}(8)$. The quark mass values quoted here indicate the offset from a critical value on a 10x10 lattice at $\beta=4.5$ defined as the average real part of the left branch real eigenmodes with $-2<\operatorname{Re}(\lambda)<-1.5$. The increase in the naive quenched errors at smaller quark mass can be directly traced 
to the increasing frequency of nearby real poles. In all cases, the MQA correlator is less noisy and closer to the full dynamical result. Statistical errors in the latter are related to autocorrelation problems, not the intrinsic noisiness of the data. Of course, for very small quark masses, we begin to see a deviation between MQA and full dynamical results, as the MQA does not contain the suppression of nontrivial topologies implicit in the zero mass full dynamical theory.

Amazingly, the MQA procedure makes it possible (to our knowledge, for the first time) to obtain sensible results by approaching the critical line from the interior of the spectral oval, corresponding to negative quark mass with the conventional definition (i.e. the "supercritical" regime with $\kappa>\kappa_{c}$ ). In the continuum, a negative quark mass may be removed by a $\gamma_{5}$ redefinition of the quark field, but on the lattice this region contains many more real modes extending farther away from the critical line (see Figs. 4,5,6), leading to extremely noisy quenched correlators (although of course the full dynamical theory is perfectly well-defined here). The plots shown in Fig(9) for the two point function of $\bar{\psi} \gamma_{5} \psi$ show that the MQA is effective in this case also, although the increased density of real modes in this regime makes the MQA less effective at extremely small quark masses- the results shown are for quark masses of $-0.15,-0.16$ and -0.185 (as before, on a $10 \times 10$ lattice at $\beta=4.5$ ), with the negative sign indicating that we are now working in the interior of the spectral oval . For small quark masses, the results in the naive quenched model are again dominated by noise, while the MQA and full dynamical results are close, with the statistical noise under control in the MQA even for quite small negative quark masses. For larger quark masses, where sensible results can be extracted from an uncorrected quenched calculation, the MQA correlators lie between the naive quenched and full dynamical results, as was the case previously in the subcritical domain. 

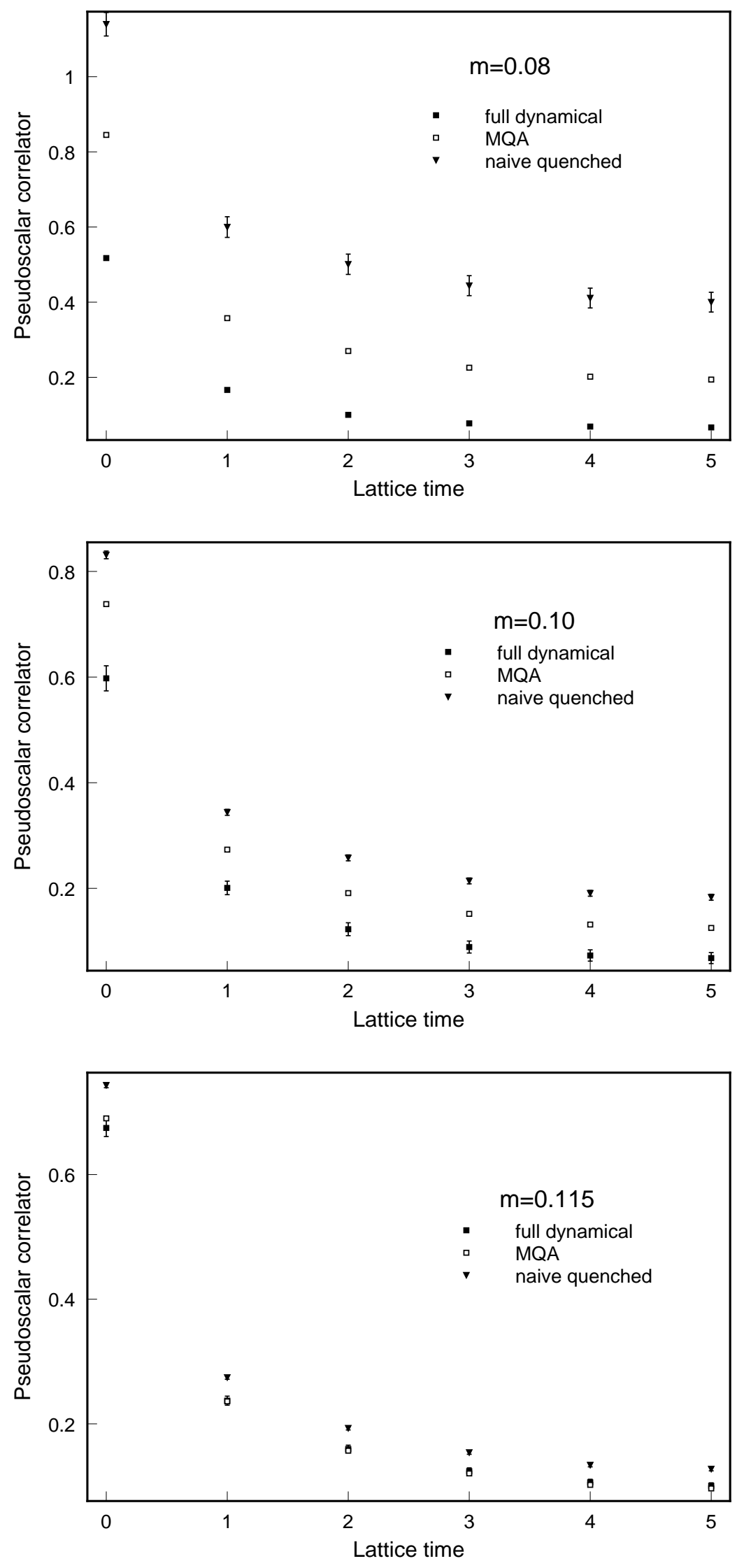

Figure 8: Pseudoscalar correlators in QED2- quenched, MQA and full dynamical 

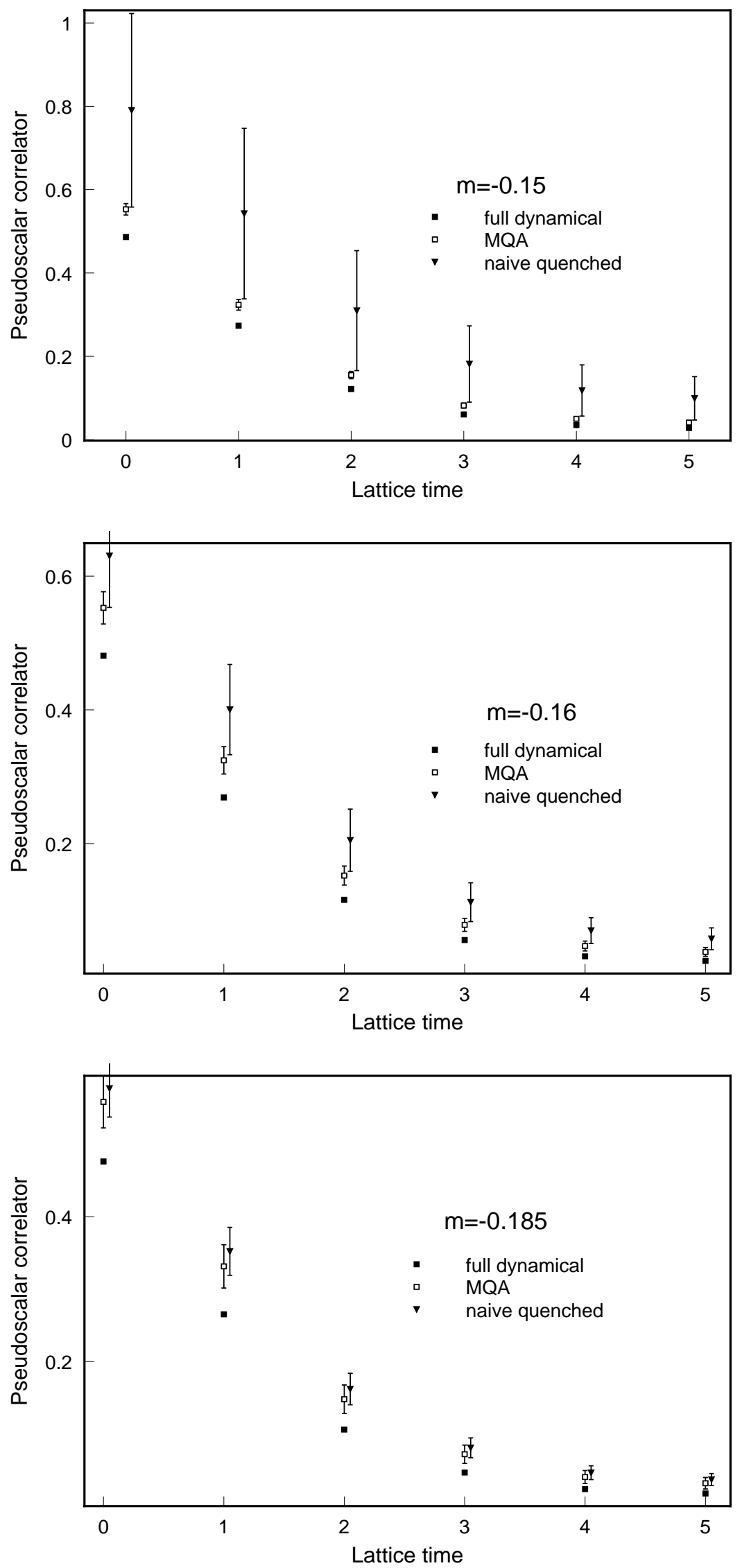

Figure 9: Supercritical pseudoscalar correlators in QED2- quenched, MQA and full dynamical 


\section{Conclusions}

The analytic arguments and numerical results presented here provide strong evidence for the interpretation of exceptional configurations as an artifact of quenched Wilson lattice theory arising from the structure of the spectrum of the discrete Wilson-Dirac matrix, and in particular from the appearance of exact real eigenmodes for particular gauge configurations close to the critical line corresponding to the chiral limit. Two dimensional QED turns out to be a particularly useful testbed for studying this problem for two reasons. First, the zero mode and topological charge structure of the theory closely mimics that of 4 dimensional QCD, at least insofar as those aspects germane to the real eigenmode problem are concerned. Secondly, extensive simulations can be performed at comparatively trivial computational cost (all of the numerical calculations reported in this paper were performed on a Pentium PC), yielding complete information about all aspects of the spectral structure. It is also possible to perform full dynamical simulations (with exact evaluation of the determinant) in this theory to test the validity of proposed modifications of the quenched approximation.

Much of the material presented in Sections 2 and 3 extends in a direct and obvious fashion to 4 dimensional QCD. There also, discrete symmetries of the Wilson-Dirac secular equation lead to the appearance of exactly real eigenmodes. The quenched functional integral as conventionally defined will be a cut function of the bare quark mass with an exceedingly complex branch structure. Pinch arguments along the lines of Section 3 can again be used to identify a subset of branch points. Unfortunately, the very useful and detailed information on the lattice spacing, lattice volume and clover coefficient dependence of the real part of the Wilson-Dirac spectrum (summarized in the histograms of Figs. 4,5, and 6) is not yet available for 4 dimensional QCD. However the results presented here for QED2 confirm in all essential details the 
picture of the zero mode problem in QCD presented in 凷. In particular, the direct comparisons of meson correlators in the conventional quenched, modified quenched (MQA), and exact dynamical simulations are a gratifying confirmation of the efficacy of the MQA method in resolving the exceptional configuration problem at finite lattice spacing.

\section{Acknowledgements}

The work of W.B. and E.E was performed at the Fermi National Accelerator Laboratory, which is operated by Universities Research Association, Inc., under contract DE-AC02-76CHO3000. The work of A.D. was supported in part by NSF grant 9322114. The work of H.T. was supported in part by the Department of Energy under grant DE-AS05-89ER 40518. We are grateful for the continued assistance of G. Hockney. 


\section{References}

[1] For a general discussion of the quenched error, see M. Golterman, heplat/9405002.

[2] A. Morel, J. Phys (paris) 48,(1987)1111; S. Sharpe, Phys. Rev. D41, (1990) 3233;

C. Bernard and M. Golterman, Phys. Rev. D46 (1992)853.

[3] K.-H. Mütter, Ph. de Forcrand, K. Schilling, and R. Sommer, in Brookhaven 1986, Lattice Gauge Theory Proceedings, p 257; M. Luescher, S. Sint, R. Sommer, P.Weisz and U. Wolff, hep-lat/9609035.

[4] W. Bardeen, A. Duncan, E. Eichten, G. Hockney and H. Thacker, Fermilab preprint, April 1997.

[5] J. Smit and J. Vink, Nucl. Phys. B286(1987)485; J. Vink, Nucl. Phys. B307(1988)549.

[6] B. Sheikholeslami and R. Wohlert, Nucl. Phys. B259(1985)572.

[7] N. K. Nielsen and B. Schroer, Nucl. Phys. B127(1977) 493.

[8] T. Banks, J. Kogut and L. Susskind, Phys. rev. D13 (1976),1043; L. Susskind, Phys. Rev. D16(1977)3031.

[9] "The Analytic S-matrix", R.J. Eden, P.V. Landshoff, D.I. Olive, and J.C. Polkinghorne, Cambridge University Press 1966, p.47 ff.

[10] A. Duncan and M. Furman, Nucl. Phys. B190, (1981) 767. 